Intensivmed 2010 • 47:9-10

DOI 10.1007/s00390-009-0132-2

Online publiziert: 30. Januar 2010

(c) Springer-Verlag 2010

\author{
U. Janssens \\ Klinik für Innere Medizin, St.-Antonius-Hospital, Eschweiler
}

\section{Ethik in der Intensivmedizin}

Hamburger Ex-Senators im Bereich des assistierten Suizids (http://www.suizidbegleitung.de; Zugriff am 03.01.2010 nicht mehr möglich, da durch ein Passwort geschützt) sind ein klares Indiz dafür, dass Patienten sich von der Medizin verlassen fühlen und andere Wege aus einem für sie nicht mehr erträglichen Leben suchen.

Sterben auf der Intensivstation ist häufig und kommt selten unerwartet. Amerikanische und europäische Daten belegen eindrucksvoll, dass dem Tod eines Intensivpatienten in mehr als 70\% der Fälle ein Behandlungsverzicht oder eine Therapielimitierung vorangeht [2].
Diesen Entscheidungen sind in der Regel sehr komplexe Diskussionen vorgeschaltet, die neben enormem medizinischem Sachverstand dem Intensivmediziner auch juristische Kenntnisse abverlangen und eine hohe soziale Kompetenz und Kommunikationsfähigkeit erfordern. Dabei spielt die Medizinethik eine zentrale Rolle. Das Wohlergehen der Patienten, das Verbot zu schaden („Primum non nocere“) und das Recht auf Selbstbestimmung (Prinzip der Autonomie) sind elementare Bestandteile einer ethisch ausgerichteten Entscheidungsfindung. chzeitig nachweisbar zunehme Anteil sehr alter Patienten auf der Intensivstation [1] stellen die Intensivmediziner vor neue Herausforderungen. Dabei spielt die altersabhängig wachsende Anzahl an Komorbiditäten eine besondere Rolle. Chronische Erkrankungen wie Herzinsuffizienz, Diabetes oder Niereninsuffizienz sind für die Prognose neben dem Alter - von herausragender Bedeutung. Die natürlichen Grenzen des Lebens werden durch eine effektive Intensivmedizin tatsächlich immer weiter verschoben - die Hochleistungsmedizin scheint keine Grenzen mehr zu kennen. Neben dem Wunsch vieler Menschen nach einem langen und möglichst gesunden Leben macht sich aber auch eine Angst vor einer inhumanen Apparatemedizin breit, die ein Sterben nicht zulässt und einen Sterbeprozess unnötig und qualvoll verlängert. Diese zum Teil sicher berechtigten Ängste werden durch Medien, Vereinigungen (z. B. Dignitas) und Einzelpersonen instrumentalisiert. Die Aktivitäten eines

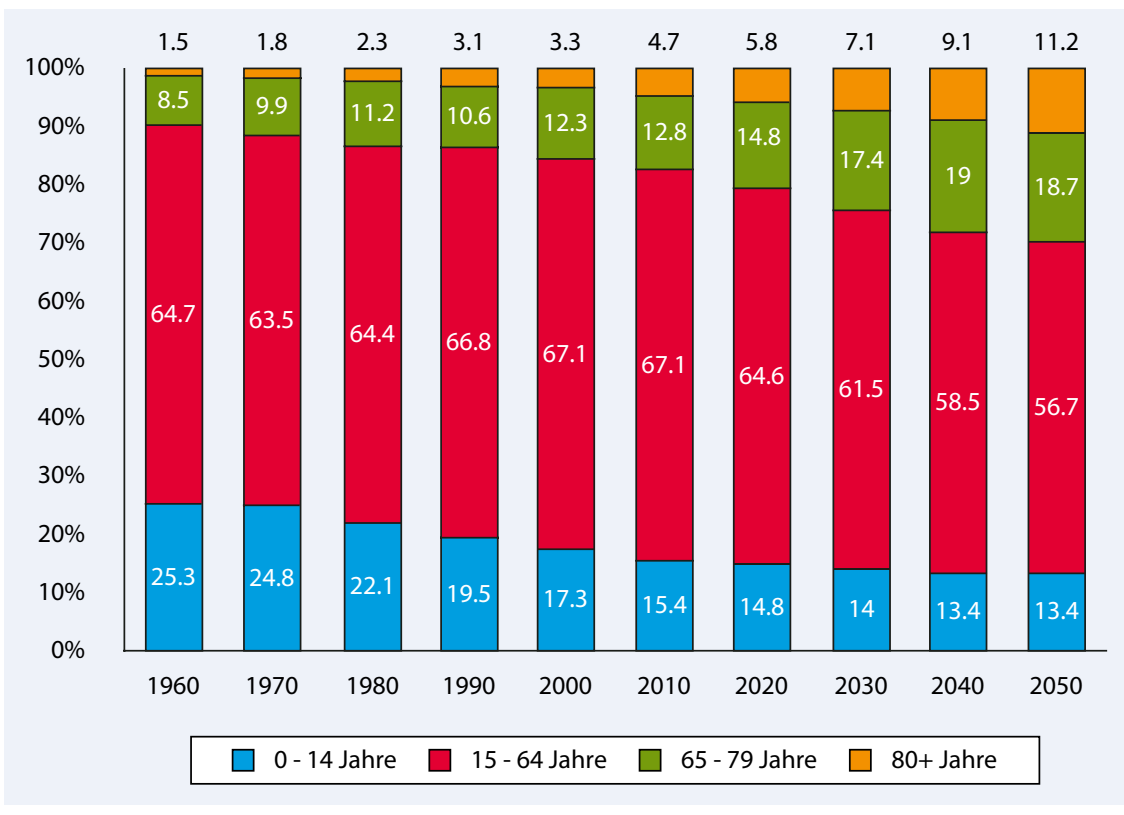

Abb. 1 A Entwicklung der Alterstruktur in der Europäischen Union zwischen 1960 und 2050. (Nach http://ec.europa.eu/employment_social/spsi/docs/social_situation/ssr2007_en.pdf, Zugriff am 12.06.2009) 
Die Beiträge der aktuellen Ausgabe beleuchten das Thema „Ethik“ sowohl theoretisch als auch praxisorientiert. P. Schmucker führt in die Thematik ein, sein Beitrag ist propädeutisch zu werten, liefert aber gleichzeitig auch praktische Beispiele. W. Druml weist auf die Bedeutung nicht nur einer neuen Terminologie, sondern auch einer neuen Sichtweise der Therapie am Lebensende hin. Der Stellenwert der Palliativmedizin wird im Beitrag von A. Valentin eindrucksvoll untermauert. Das im vergangenen Jahr verabschiedete Gesetz zur Änderung des Betreuungsrechts weist der Patientenverfügung eine besondere und neue Rolle zu. A. Simon geht in seinem Beitrag auf das neue Gesetz und seine Bedeutung für die Intensiv- und Notfallmedizin ein. Die Angehörigen von Intensivpatienten sind gerade bei nicht kompetenten, bewusstseinsgestörten Patienten für die Entscheidungsabläufe unabdingbar und benötigen umgekehrt Unterstützung durch das gesamte Behandlungsteam. Der Beitrag zur Angehörigenkonferenz von U. Janssens zeigt Wege auf, wie eine solche Besprechung zu planen und durchzuführen ist. Viele der genannten Aspekte werden durch A. Wallner abschließend zusammengefasst: Die Organisation medizinischer Entscheidungen am Lebensende darf kein Zufall sein, sie ist genau zu planen und elementarer Bestandteil der Behandlungsqualität auf der Intensivstation.

Als Herausgeber dieses Themenheftes darf ich den Autoren für ihre exzellenten Beiträge danken.

Gleichzeitig lade ich als Sprecher der Sektion „Ethik“ der Deutschen Gesellschaft für Intensivmedizin und Notfallmedizin zur aktiven Mitarbeit ein. Als Intensivmediziner müssen wir eine führende Rolle in ethischen Diskussion und Fragestellungen einnehmen und damit auch gestaltend in gesellschaftliche Prozesse eingreifen.
„Wo das Bewusstsein schwindet, dass jeder Mensch uns als Mensch etwas angeht, kommen Kultur und Ethik ins Wanken. Das Fortschreiten zur entwickelten Inhumanität ist dann nur noch eine Frage der Zeit."

Albert Schweitzer (1875-1965), deutschfranzösischer Arzt, Theologe, Musiker und Kulturphilosoph, 1952 Friedensnobelpreis

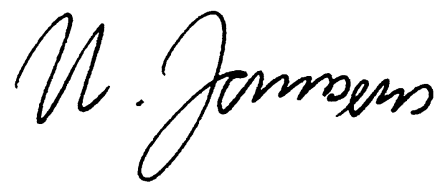

Uwe Janssens

\section{Korrespondenzadresse}

\section{Prof. Dr. U. Janssens}

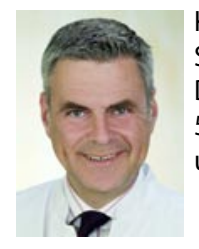

Klinik für Innere Medizin, St.-Antonius-Hospital Dechant-Deckers-Str. 8, 52249 Eschweiler uwe.janssens@sah-eschweiler.de

\section{Literatur}

1. Bagshaw SM, Webb SA, Delaney A et al (2009) Very old patients admitted to intensive care in Australia and New Zealand: a multi-centre cohort analysis. Crit Care 13:R45 (doi:10.1186/cc7768)

2. Sprung CL, Cohen SL, Sjokvist P et al (2003) Endof-life practices in European intensive care units: the Ethicus Study. JAMA 290:790-797
Telemedizin zum Anfassen

Zu den Highlights der CeBIT (02.-06.03.10, Hannover) gehört in diesem Jahr für Ärzte und Gesundheitspersonal die Sonderschau FutureCare innerhalb der TeleHealth, die in Halle 9 stattfindet.

Die Vernetzung unterschiedlicher medizinischer Bereiche wie Prävention, Diagnose, Röntgenaufnahmen, Therapie, Nachsorge etc. verlangt eine reibungslose Weitergabe von unstrukturierten Informationen und Daten und ist heute in der Medizin von entscheidender Bedeutung. Anhand verschiedener realer Anwendungen wird auf der FutureCare gezeigt, wie sich Ärzte und weitere beteiligte Berufsgruppen in der Zukunft durch eben dieses vernetzte Arbeiten noch mehr um den Patienten kümmern können. „Für fast jede Fachgruppe gibt es große Möglichkeiten für einen Einsatz von Telemonitoring zum Vorteil von Patienten, und die Investitionen liegen nahe null", meint Professor Harald Korb, Beiratsvorsitzender der TeleHealth.

Verschiedene Rundgänge zu Themen wie dem Einsatz telemedizin-gestützter Tertiärprävention, integrierter Versorgung in Kliniken oder Nutzung elektronischer Notfalldaten in einem akuten Notfall zeigen, wie Daten über den gegenwärtigen gesundheitlichen Zustand des betreffenden Menschen Auskunft geben und wie diese Daten für weitere Entscheidungen für eine optimale gesundheitliche Versorgung rechtzeitig zur Verfügung stehen.

\section{Kostenlose E-Tickets für Leser} der „Ärzte Zeitung“ Für die CeBIT steht der „Ärzte Zeitung“ als Medienpartner der begleitenden Messe TeleHealth ein Kontingent kostenloser E-Tickets zur Verfügung, die Haus- und Fachärzte, aber auch Kliniker und andere Heilberufler in Anspruch nehmen können. Die Karten berechtigen zum einmaligen Besuch von CeBIT und TeleHealth.

Die Tickets sind registrierungspflichtig. Ein Link auf www.aerztezeitung.de im Kasten „TeleHealth / CeBIT 2010" in der Navigationsspalte rechts führt zur Registrierung. Nach Registrierung wird das Ticket an die angegebene E-Mail-Adresse versandt. Der Ausdruck genügt für den Eintritt.

Quelle: Ärzte Zeitung, www.aerztezeitung.de 